\title{
Investigation of the initial stages of spark-plasma sintering of Si-Ge based thermoelectric materials
}

\author{
M. V. Dorokhin, I. V. Erofeeva, Yu. M. Kuznetsov, M. S. Boldin, A. V. Boryakov, A. A. Popov, \\ E. A. Lantsev, N. V. Sakharov, P. B. Demina, A. V. Zdoroveyshchev, V. N. Trushin \\ N. I. Lobachevsky State University of Nizhniy Novgorod, Gagarin ave. 23/3, 603950, Nizhniy Novgorod, Russia \\ dorokhin@nifti.unn.ru, irfeya@mail.ru, yurakz94@list.ru, boldin@nifti.unn.ru, Boryakov@phys.unn.ru, \\ popov@nifti.unn.ru, elancev@nifti.unn.ru,nvsaharov@nifti.unn.ru, Demina@phys.unn.ru, \\ zdorovei@gmail.com, trushin@phys.unn.ru
}

DOI 10.17586/2220-8054-2018-9-5-622-630

\begin{abstract}
Thermoelectric materials based on a mixture of Ge-Si nanopowders were fabricated and investigated. The materials were obtained by spark plasma sintering technique using the modes corresponding to the initial stages of sintering of the powder particles. The possibility for controlling the electrical characteristics of materials (type and magnitude of conductivity, the Seebeck coefficient) by varying the sintering parameters was shown. It was found that the analysis of electrical characteristics allows one to draw conclusions about the degree of mixing for silicon and germanium in the sintered material.
\end{abstract}

Keywords: thermoelectric power sources, Seebeck effect, nanostructures, spark plasma sintering.

Received: 5 April 2018

Revised: 3 August 2018

\section{Introduction}

Silicon- and germanium-based semiconductors are widely known high-temperature thermoelectric materials [1]. The main characteristic parameter for thermoelectric materials is the dimensionless thermoelectric figure of merit - ZT, which in turn depends on such macroscopic parameters as thermal conductivity $\kappa$, electrical conductivity $\sigma$ (or resistivity $\rho=1 / \sigma$ ), and Seebeck coefficient $\alpha=U_{t e} / \Delta T$, where $U_{t e}$ is the Seebeck voltage, $\Delta T$ is the temperature difference between the "hot" and "cold" ends of the material:

$$
Z T=\frac{\alpha^{2} \sigma}{\varkappa} T \text {. }
$$

As a rule, in every single material, these parameters are not independent of each other: an increase in electrical conductivity leads to a decrease in the thermoelectric voltage, etc.

Modern trends of the thermoelectric technology are associated with the fabrication of materials with ultrafinegrained polycrystalline structure, which provides low thermal conductivity while maintaining both high Seebeck coefficient and electrical conductivity [2]. In addition, unlike that of the single crystals, the figure of merit for polycrystalline thermoelectric materials is determined by the grain size, grain boundary properties, the degree of $\mathrm{Ge}$ and Si intermixing in a solid solution [3]. Controlling these parameters would ensure the control of the material properties for fabrication of efficient thermoelectric energy converters.

In the present paper, we report on the results of the investigation of $\mathrm{Si}_{0.8} \mathrm{Ge}_{0.2}$ thermoelectric materials. The selection of 0.8 vs 0.2 composition was determined by known literature data [4-6], in which it was demonstrated that such composition provides low thermal conductivity accompanied with relatively high Seebeck voltage (thus it allows one to obtain high ZT value). The $\mathrm{Si}_{0.8} \mathrm{Ge}_{0.2}$ was obtained by the spark plasma sintering (SPS) method using Ge and Si powders. The SPS method provides ample opportunities for controlling the polycrystalline structure parameters [7]. At the same time, the interrelation of the sintering modes, the parameters of the polycrystalline structure and the resulting thermoelectric characteristics is not trivial. To the best of our knowledge, there are no general models describing such a relationship. The influence of sintering conditions on the materials' properties is very often studied by experimental methods only, using the fabrication of a rather big set of samples in various technological modes [3-9].

We present the results for the experimental study of the initial stages of the fabrication of $\mathrm{Si}-\mathrm{Ge}$ based thermoelectric materials by means of spark plasma sintering method. The evolution of the material's grain structure within the framework of varied sintering modes, as well as the influence of the grain structure on the thermoelectric characteristics were studied. 


\section{Experimental techniques}

The investigated samples were sintered from the Ge and Si powders using the DR SINTER model SPS625 spark plasma sintering system. As raw material for powders, ingots of single-crystal Ge and Si were used. The source of Ge was an ingot doped with the donor impurity (Sb) to a concentration of $\sim 10^{18} \mathrm{~cm}^{-3}$. As for the $\mathrm{Si}$, an ingot doped with an acceptor impurity (B) was used. The acceptor concentration was $\sim 2 \times$ $10^{15} \mathrm{~cm}^{-3}$. The materials with different types of doping were chosen in order to estimate the degree of mixing by performing conductivity measurements. At this stage, the composition of the $\mathrm{Si}-\mathrm{Ge}$ material was assessed, the latter corresponded to the $\mathrm{Si}_{0.8} \mathrm{Ge}_{0.2}$ atomic composition for all the samples studied.

The Ge and Si ingots were preliminarily milled and then mechanically activated using a Fritch Pulverisette 7 planetary mill with a ceramic container volume of $500 \mathrm{ml}$ and a size of grinding balls $d \approx 10 \mathrm{~mm}, m \approx 5 \mathrm{~g}$. The average crystallite size in the powder was $100 \mathrm{~nm}$ to $50 \mu \mathrm{m}$, depending on the milling modes. The grain size was monitored by means of a scanning electron microscope. Complete information on the parameters of the powders is presented in Table 1. The mass of the initial feed material in all cases was $30 \mathrm{~g}$. The grinding time was 1 or 6 hours. The increase in the grinding time provided a reduction in the average grain size of the obtained powders to a value on the order of hundred nanometers.

TABLE 1. Parameters of milling and sintering

\begin{tabular}{|c|c|c|c|c|}
\hline No. & $\begin{array}{c}\text { Powder } \\
\text { grain size }\end{array}$ & Milling modes & Sintering modes & $\begin{array}{c}\text { Grain size } \\
\text { after sintering }\end{array}$ \\
\hline 1 & $5-20 \mu \mathrm{m}$ & $\begin{array}{l}\text { Pulverisette 7, } \\
1 \text { hour milling }\end{array}$ & $\begin{array}{l}\text { Linear heating to } 970{ }^{\circ} \mathrm{C}, \\
8.2 \mathrm{kN} \text { pressure, } 400 \mathrm{sec} .\end{array}$ & $5-20 \mu \mathrm{m}$ \\
\hline 2 & $5-20 \mu \mathrm{m}$ & $\begin{array}{l}\text { Pulverisette 7, } \\
1 \text { hour milling }\end{array}$ & $\begin{array}{l}\text { Linear heating to } 970{ }^{\circ} \mathrm{C}, \\
8.2 \mathrm{kN} \text { pressure, } 370 \mathrm{sec} .\end{array}$ & $5-20 \mu \mathrm{m}$ \\
\hline 3 & $0.1-5 \mu \mathrm{m}$ & $\begin{array}{l}\text { Pulverisette 7, } \\
6 \text { hours milling }\end{array}$ & $\begin{array}{l}\text { Linear heating to } 930{ }^{\circ} \mathrm{C}, \\
8.2 \mathrm{kN} \text { pressure, } 370 \mathrm{sec} .\end{array}$ & $0.1-5 \mu \mathrm{m}$ \\
\hline 4 & $0.1-15 \mu \mathrm{m}$ & $\begin{array}{l}\text { Pulverisette 7, } \\
6 \text { hours milling }\end{array}$ & $\begin{array}{c}\text { 2) Same as for sample } 3 ; \\
22 \mathrm{kN} \text { pressure, } 700 \mathrm{sec} .\end{array}$ & $100-400 \mu \mathrm{m}$ \\
\hline 5 & Control sample $-p$-type Si:B plate $\left(p \sim 2 \times 10^{15} \mathrm{~cm}^{-3}\right)$ \\
\hline
\end{tabular}

The resulting powder was placed into a graphite mold and subjected to sintering in various modes (data are given in Table 1). Two types of samples were fabricated. Samples 1, 2 and 3 were sintered in one step at a constant heating rate to the limiting temperature of $930-970{ }^{\circ} \mathrm{C}$ (Table 1). Sample 4 was fabricated in two steps: within the first step, the sample was heated at a constant rate to $T=930{ }^{\circ} \mathrm{C}$, thus preliminary sintering and compacting of the powders were performed. Within a second step, the pre-compacted powder was placed in a new (larger) mold and further sintered at a constant heating rate to a higher temperature of $T=1200{ }^{\circ} \mathrm{C}$; the latter point corresponds to the melting of the $\mathrm{Si}-\mathrm{Ge}$ material. Thus, Samples 1,2 and 3, fabricated in one step, correspond to the initial stages of sintering, during which the powder compaction takes place and Ge and Si mutual diffusion starts at the grain boundaries. Sample 4 represents the next sintering step, during which a solid solution of $\mathrm{Si}_{1-x} \mathrm{Ge}_{x}$ and a new polycrystalline structure are formed. The control Sample 5 was the $p$-Si plate with the same doping level as the initial material for the silicon powder. This sample was studied for the purpose of monitoring and comparing the results.

The crystalline structure and phase composition of the deposited films were studied using a Shimadzu 7000 $\mathrm{X}$-ray diffractometer (Japan), the $\mathrm{CuK} \alpha$ radiation with a focal spot size of $0.4 \times 12 \mathrm{~mm}^{2}$ was used. The illumination area size of the surface was $1.2 \times 1.8 \mathrm{~mm}^{2}$. Phase analysis of the samples was carried out on the basis of the DIFFRAC.EVA software complex, using a powder database PDF-2. Based on the analysis, conclusions were drawn about the phase composition.

The composition of the resulting material was determined by X-ray microanalysis using the Jeol JSM-IT300LV scanning electron microscope with the Oxford Instruments X-MaxN 20 energy dispersive attachment (equipment of the "New Materials and Resource-Saving Technologies" Center). An accelerating voltage of $20 \mathrm{kV}$ was used; the probe current was several nA. The measurements were carried out in a high vacuum mode. When determining the composition and constructing maps of the distribution of elements over the surface, the $\operatorname{SiK} \alpha$, GeK $\alpha$, and GeL $\alpha$ lines were used. The composition was calculated by the method of fundamental parameters implemented 
on the basis of the software of the microanalysis system. The determined Si-Ge composition corresponded to the calculated and prepared $\mathrm{Si}$ and $\mathrm{Ge}$ atomic ratio of the material $\left(\mathrm{Si}_{0.8} \mathrm{Ge}_{0.2}\right)$.

Sintered bulk samples were cut into plates with dimensions (length/width/height) of $10 \times 5 \times 3 \mathrm{~mm}$. One of the plate surfaces was subjected to mechanical abrasion and polishing. To measure the thermoelectric characteristics, metallic ohmic contacts were deposited on the surface of the samples. The polished surface of the samples was examined by scanning electron microscopy using the Jeol JSM-IT300LV device. Maps of the elemental distribution along the surface of structures were constructed on the obtained electron-microscopic images.

The thermoelectric characteristics were measured for each structure at temperatures ranging from 50 to $350{ }^{\circ} \mathrm{C}$. The edges of the sample were placed on two independent furnaces. A constant temperature difference of $\pm 10 \mathrm{~K}$ was created between the ends of the sample. As a result, a Seebeck voltage appeared at the ends of the sample, this voltage was measured with a voltmeter. The value of the Seebeck coefficient was calculated as:

$$
\alpha=\frac{1}{2}\left(\frac{U_{+}}{+10 \mathrm{~K}}+\frac{U_{-}}{-10 \mathrm{~K}}\right)
$$

where $U_{+}\left(U_{-}\right)$- is the thermoelectric voltage for arbitrary positive (negative) temperature difference (taking into account the sign). As the measurement temperature, an average value between the temperatures of the "hot" and "cold" ends was used. The measurements were carried out under vacuum with a residual vapor pressure of about $10^{-3}$ Torr.

At zero temperature difference, the electrical resistance of the sample was also measured as a function of temperature. Taking into account the known geometric dimensions of the sample, the resistivity of each structure was calculated.

\section{The structure and composition measurements}

The X-Ray diffraction (XRD) spectra of all fabricated samples are shown at Fig. 1. The spectra represent the set of peaks corresponding to $\mathrm{Si}, \mathrm{Ge}$ and $\mathrm{Ge}-\mathrm{Si}$ solid solutions. We first should note the lower intensity of XRD peaks revealed for the Sample 4. This is the evidence of the lower crystalline quality of the sample as compared with Samples 1-3. For the Sample 3 (fabricated at the lowest temperature of $930{ }^{\circ} \mathrm{C}$ ) the set of peaks is attributed with the phases of pure silicon, pure germanium and $\mathrm{Si}_{1-x} \mathrm{Ge}_{x}$ solid solution. This allows us to suggest that sintering at the Sample 3 modes (Table 1) did not lead to significant mixing of Ge and Si particles (although the mixing process was initialized). In Samples 1 and 2, the Ge-related XRD peak was not revealed although Si and $\mathrm{Ge}-\mathrm{Si}$ related peaks are still present at the spectra. We suggest that increase of the sintering temperature for the Sample 1 and 2 (to $970{ }^{\circ} \mathrm{C}$ ) has enhanced the sintering process thus the relative content of Ge-Si solid solution was increased whereas Ge content was decreased to the undetectable value.

However, the presence of Si-related peaks evidences that unmixed Si particles remain in the bulk of the material. Finally, in the Sample 4 (which was sintered at $1200{ }^{\circ} \mathrm{C}$ ) no $\mathrm{Si}$ and Ge-related peaks were revealed. Instead, a set of peaks related with different atomic composition of $\mathrm{Si}_{1-x} \mathrm{Ge}_{x}$ solid solution are detected. We believe that the mixing of $\mathrm{Si}$ and Ge particles occurred for the high-temperature grown samples and new atomic structure with two phases of $\mathrm{Si}_{1-x} \mathrm{Ge}_{x}$ solid solution with different $\mathrm{Ge}-\mathrm{Si}$ composition was formed.

Figure 2 shows the enlarged images of the polished surface of $\mathrm{Si}-\mathrm{Ge}$ samples obtained by a scanning electron microscope. A grain structure can be revealed, the grain dimensions correspond to the characteristic grain sizes of the original powder. In particular, a higher grinding time for Sample 3 leads to a registration of smaller grains (Fig. 2(c)) as compared to Samples 1 and 2 (Fig. 2(a) and (b), respectively). In addition, some pores were detected on the surface of Samples 1-3 are (dark areas on microscopic images), the presence of the pores is believed to be due to the selected modes of sintering, i.e. a combination of pressure and heating. Modification of the sintering regime (changing the algorithm for applying pressure to the mold) leads to a reduction in the number of pores for Sample 1 as compared to Sample 2 (Figures 2a and b, respectively). This in turn corresponds to a greater shrinkage of the powder during sintering of Sample 1. Note that the Sample 4, obtained with the melting of the powder, is characterized by a grain structure that is fundamentally different from the previous ones.

For Sample 4, a significantly increased grain size was revealed, that does not correspond to the grain size of the original powder; additionally, significantly fewer pores were noted in its structure (Fig. 2(d)). These characteristics are associated with the features of sintering the material in the melting mode. We note that the single-crystal $p$-Si plate is characterized by an atomically smooth homogeneous surface (data not shown).

Both the data on the grain structure and XRD spectra can be supported by the results of the composition investigations, which were performed by the energy-dispersive X-ray spectroscopy in the microanalysis mode. Fig. 3 shows maps of the lateral distribution of Ge and Si for all the investigated samples. For better authenticity 


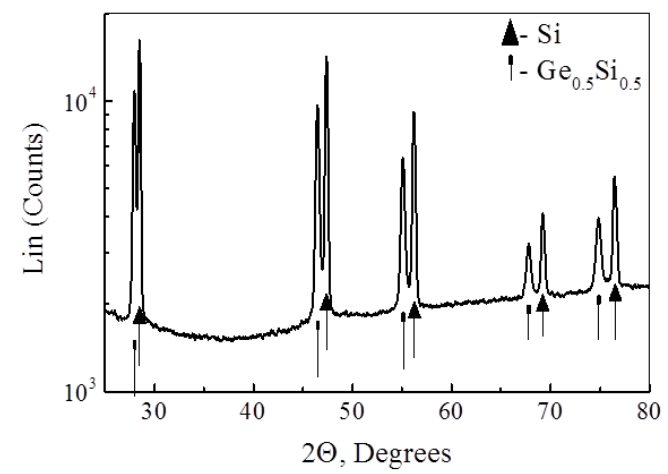

(a)

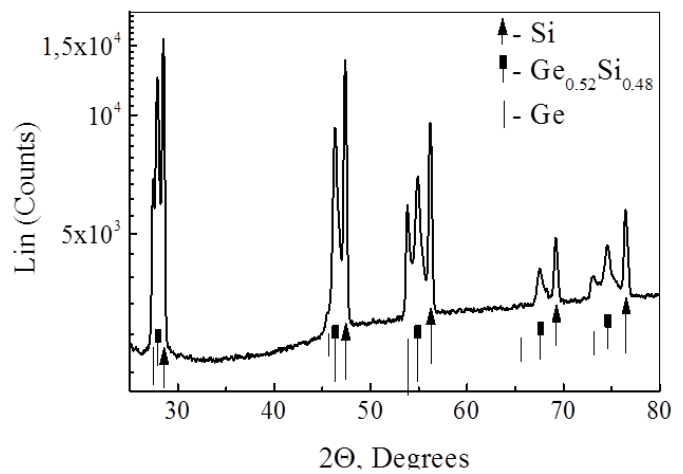

(c)

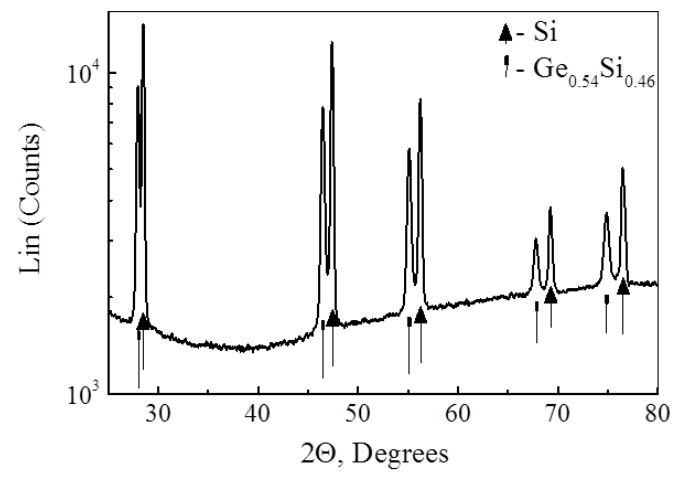

(b)

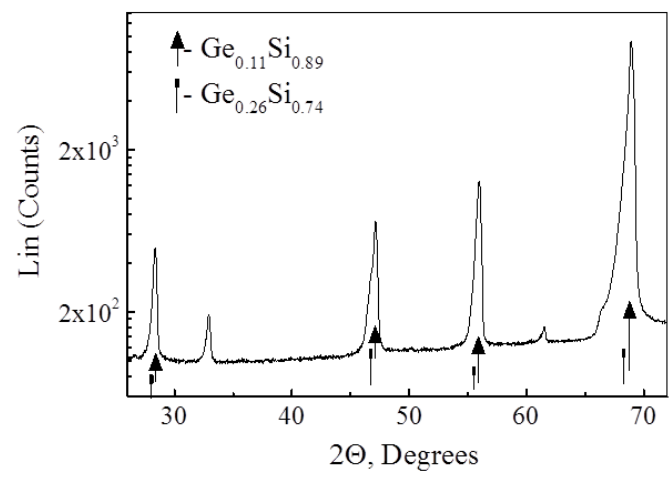

(d)

FIG. 1. XRD spectra of investigated samples (a) Sample 1; (b) - 2; (c) - 3; (d) - 4. Arrows indicate the position of diffraction lines for the different $\mathrm{Ge}, \mathrm{Si}$ and $\mathrm{Si}_{1-x} \mathrm{Ge}_{x}$ phases. The Ge content in the $\mathrm{Si}_{1-x} \mathrm{Ge}_{x}$ phase was calculated from the lattice constant parameters revealed from the peak position, the $x$ value was derived from formula given in [10]. The revealed $\mathrm{Si}_{1-x} \mathrm{Ge}_{x}$ phase is indicated at each graph. Two unmarked peaks at Fig. 1(d) could not be attributed to any of the peaks in the database

of the results, additional measurements in the regions corresponding to the maxima of the Ge and Si concentrations were also made.

From the data presented in the Fig. 3, one can reveal the regions with the composition close to 100 at. $\%$ $\mathrm{Si}$, and regions with a composition close to 100 at. \% Ge can (Fig. 3(a-c)). The size of these regions correspond exactly to the size of the grains detected in electron-microscopic studies; the area ratio of $\mathrm{Si}$ and $\mathrm{Ge}$ grains corresponds to the $\mathrm{Si}_{0.8} \mathrm{Ge}_{0.2}$ composition. Such results are in good agreement with the XRD phase analysis; they confirm the absence of significant mixing of Ge and Si atoms in the sintering modes 1-3 (modes without to melting of the material). A similar trend is maintained for Sample 3, with a smaller grain size (Fig. 3(c)). Thus, Samples 1-3 represent a two-phase system of mostly unmixed Ge and Si. When sintering in the melting mode (Sample 4), a mixing of $\mathrm{Ge}$ and $\mathrm{Si}$ takes place, which is shown by a rather uniform distribution of $\mathrm{Si}$ and Ge over the sample surface (Fig. 3(d)).

We note regions with locally high Ge content, the location of these regions corresponds to the boundaries of large grains. One can draw a conclusion that Ge atoms are accumulated in the grain boundary region. The latter result is again in the good agreement with XRD phase analysis (Fig. 1(d)) where two phases with the different Ge content were revealed. Thus, we can note a fundamental difference between the properties of Sample 4 and Samples 1-3: Sample 4 is a GeSi solid solution characterized by a large $(100 \mu \mathrm{m})$ grain structure.

\section{Thermoelectric properties measurement}

Figure 4 shows the temperature dependences of the Seebeck coefficient for all investigated samples, as well as for the $p$-Si plate.

The sign of the Seebeck coefficient measured for the Samples 1-3, as well as for the $p$-Si plate is negative over the entire temperature range, i.e. the potential of the "hot" end of the sample is lower than that of the "cold" end. 


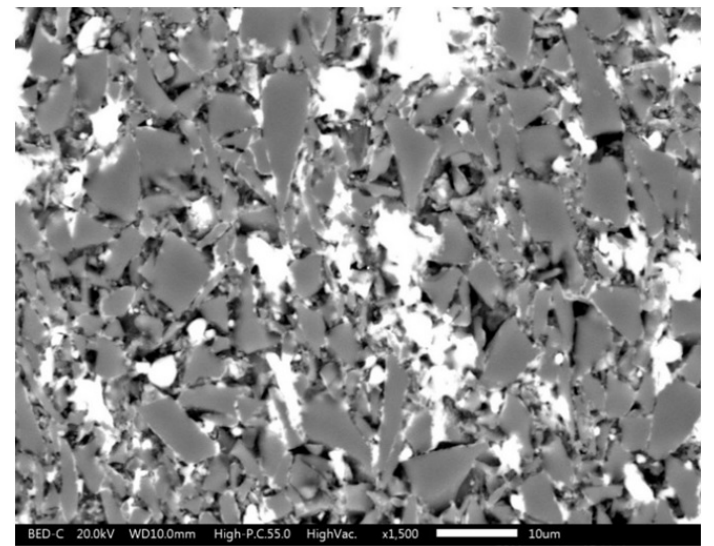

(a)

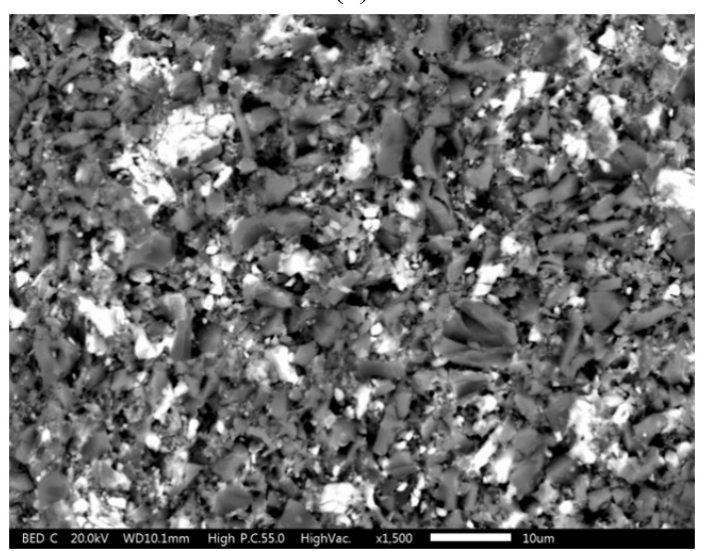

(c)

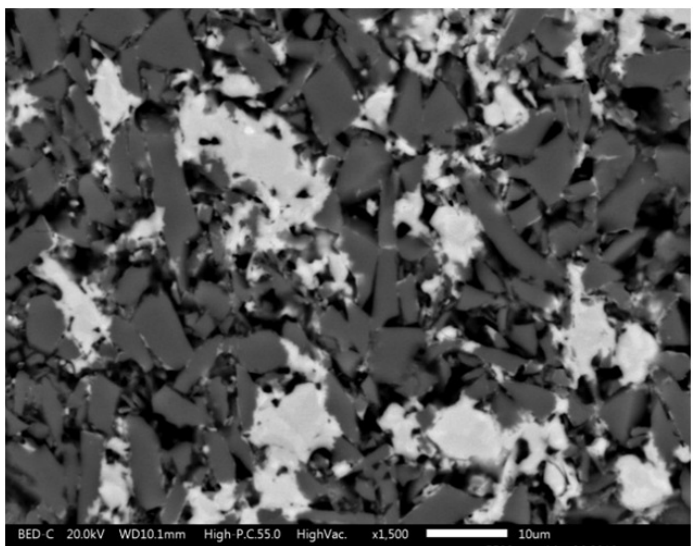

(b)

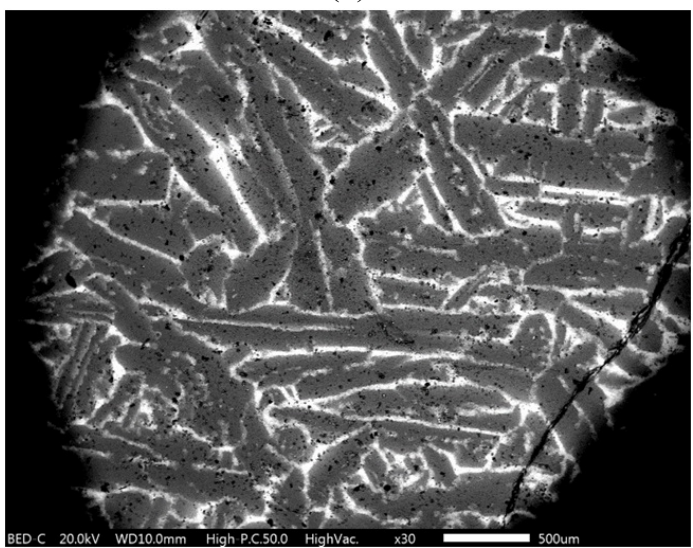

(d)

FIG. 2. The image of the polished surface of Samples $1-4$ (a - d, respectively) obtained by a scanning electron microscope in the regime of back-reflected electrons

This situation corresponds to $p$-type conductivity of the samples. The absolute value of $\alpha$ decreases monotonically with increasing temperature. The largest absolute value of $\alpha$ is $\sim 0.5 \mathrm{mV} / \mathrm{K}$, the smallest one is $\sim 0.15 \mathrm{mV} / \mathrm{K}$ (at a temperature of $350{ }^{\circ} \mathrm{C}$ ). No significant differences between the $\alpha(T)$ dependences for Samples $1-3$ were revealed. For the Sample 4, the sign of $\alpha$ coefficient is positive, which corresponds to the electronic type of conductivity. The value of $\alpha$ is $\sim 0.1 \mathrm{mV} / \mathrm{K}$ at $50{ }^{\circ} \mathrm{C}$ and it slightly decreases in the absolute value with increasing measurement temperature.

Figure 5 shows the temperature dependences of the resistivity of the investigated samples. For Samples 1-3, as well as for $p$-Si plate, one can note the semiconductor type of the $\rho(T)$ dependence: there is an exponential decrease of resistivity with increasing temperature. Curves 1-3 and 5 demonstrate similar behavior with the only difference in the resistivity values. We note a sharper temperature dependence of the resistivity of Samples $1-3$ as compared with the control structure. At a temperature of $50^{\circ} \mathrm{C}$, the resistivity of Samples $1-3$ is almost two orders of magnitude higher than for the $p$-Si (Fig. 5, curves 1-3 and curve 5, respectively). At $350{ }^{\circ} \mathrm{C}$, the resistivity values of Samples 1-3 and the control sample differ only by a factor of 2 . For sample 4, which is characterized by $n$-type conductivity, a significantly lower value of the resistivity $(\sim 0.006 \Omega \times \mathrm{m})$ was obtained. This value varies slightly over temperatures ranging $50-350{ }^{\circ} \mathrm{C}$ (Fig. 5, curve 4). Unlike Samples 1-3, the resistivity of Sample 4 is by two orders of magnitude lower than that of $p$-Si plate.

Using the Seebeck coefficient and the resistivity measurements, the values of the thermoelectric power factor were calculated from the formula:

$$
p_{T E}=\frac{\alpha^{2}}{\rho} .
$$

The temperature dependences of the power factor are shown at Fig. 6 . The largest values $(\sim 2 \times$ $10^{-6} \mathrm{~W} / \mathrm{m} \times \mathrm{K}^{2}$ ) are characteristic for the Sample 4 . 


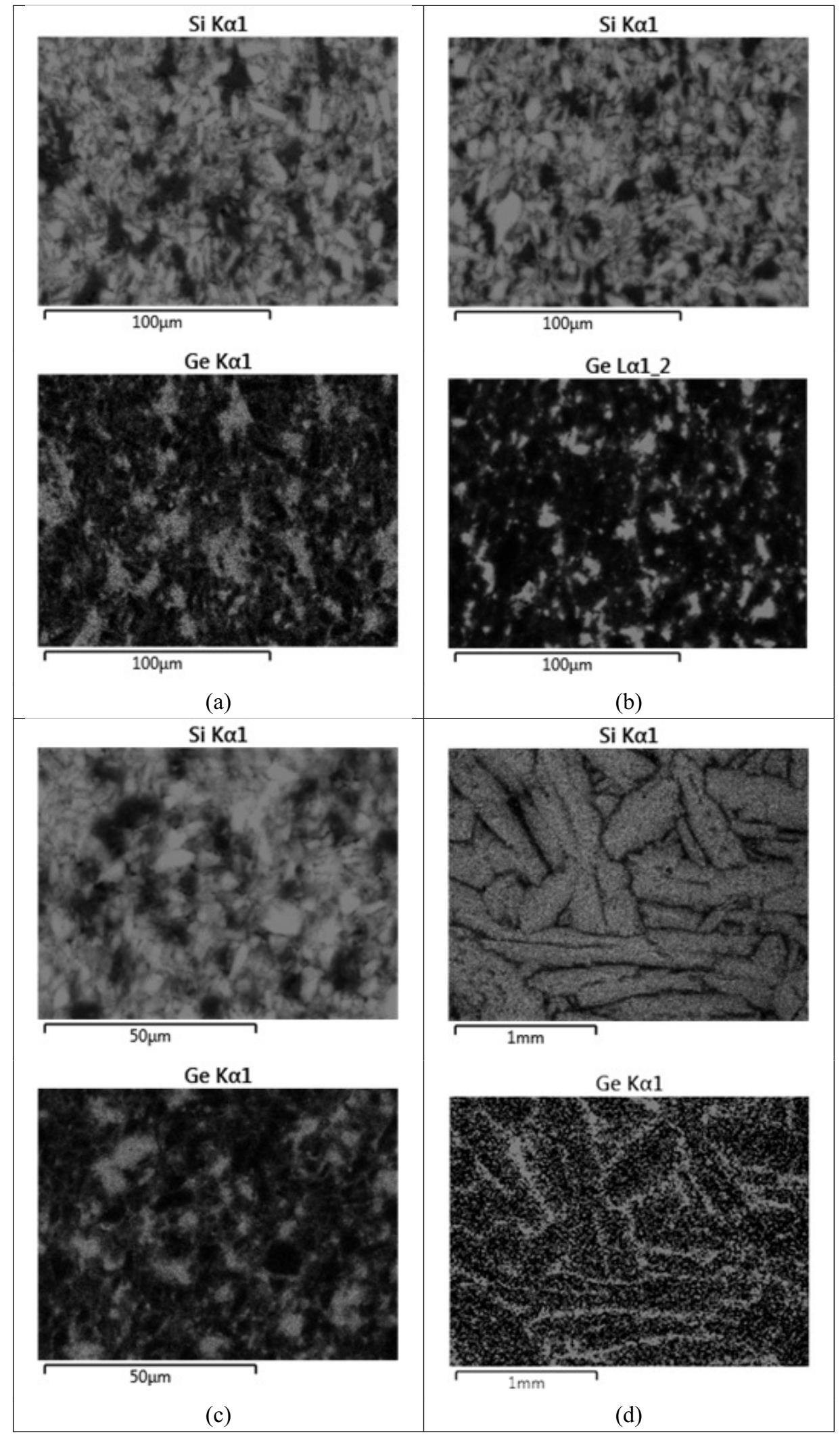

FIG. 3. Maps of the Ge and Si distribution along the polished surface of the Samples $1-4$ (a - d, respectively) 


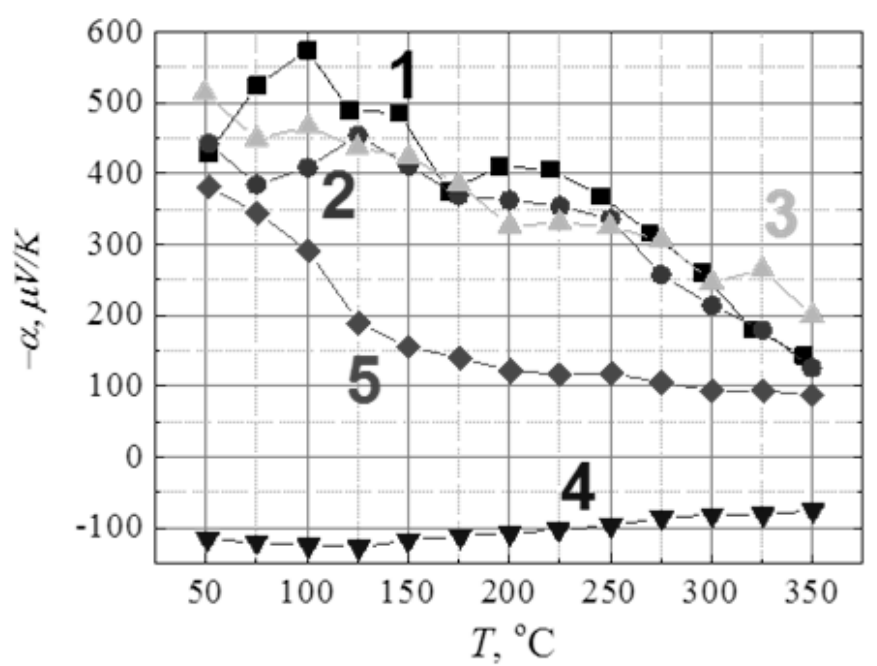

FIG. 4. Temperature dependences of the Seebeck coefficient for Samples 1 (curve 1), 2 (curve 2), 3 (curve 3), 4 (curve 4), $p$-Si (curve 5)

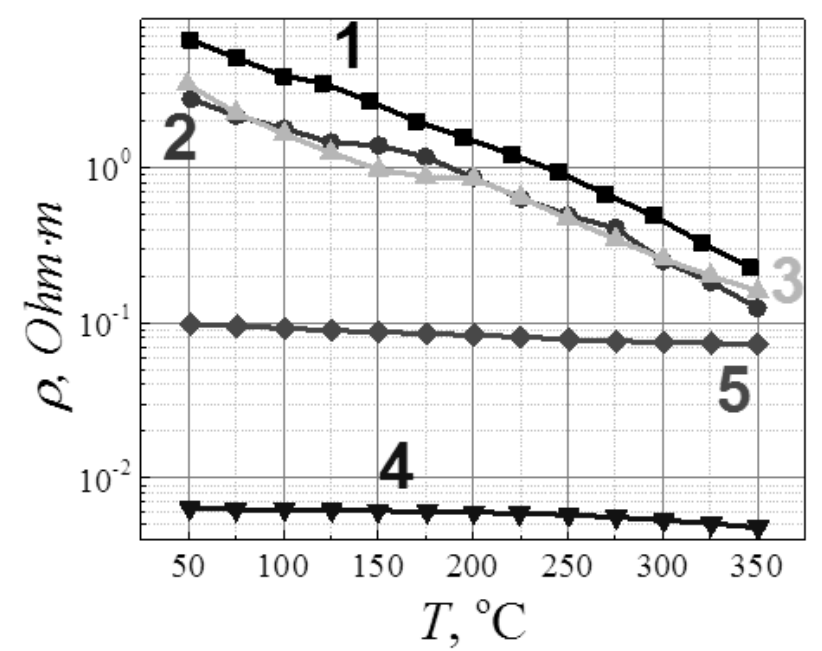

FIG. 5. Temperature dependences of the resistivity for Samples 1 (curve 1), 2 (curve 2), 3 (curve 3), 4 (curve 4), $p$-Si (curve 5)

The power factor values of Samples 1-3 increase monotonically with increasing measurement temperature, but do not exceed $2 \times 10^{-7} \mathrm{~W} / \mathrm{m} \times \mathrm{K}^{2}$. For the control structure, the strongest temperature dependence of the power factor was obtained, while the value of $p_{T E}$ decreases significantly above $100{ }^{\circ} \mathrm{C}$, and the maximum values are somewhat lower than the values obtained for Sample 4.

\section{Discussion}

First of all, we emphasize that the fabrication technology for Samples 1-3 correspond to the initial stages of Ge and Si sintering, during which the powder is compressed and mutual diffusion processes is started, however no mixing takes place yet. Such material is a combination of Ge and Si particles, whose grain structure is determined by the properties of the initial powders. Sample 4 represents the subsequent stages of sintering, during which the material is mixed and a new grain structure is formed. In this case, the properties of grain structure are determined by the sintering modes. The revealed features of thermoelectric characteristics are thus associated with the features of the grain structure formed for each sample.

For the control structure, the resistivity and the Seebeck coefficient are determined by the intrinsic material properties, in particular by the level of acceptor doping. The calculated values of the $p$-Si power factor are typical 


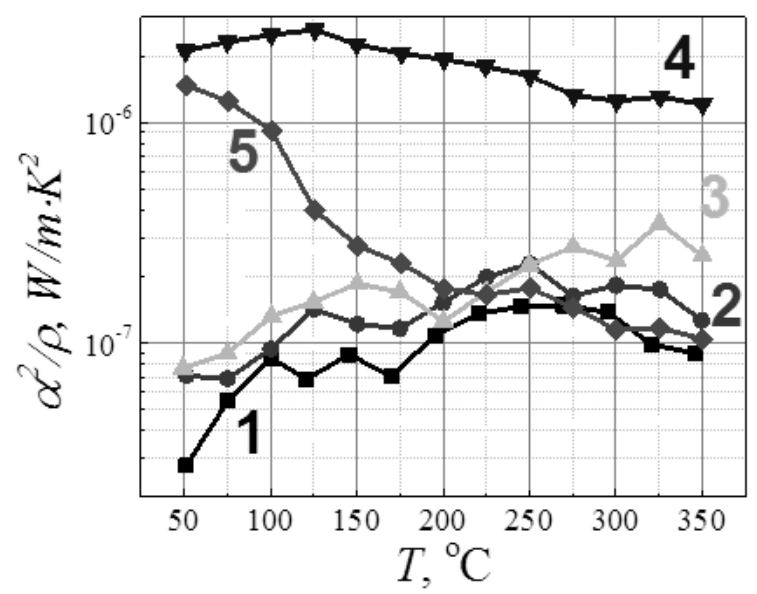

FIG. 6. Temperature dependences of the power factor calculated according to formula (2) for Samples 1 (curve 1), 2 (curve 2), 3 (curve 3), 4 (curve 4), $p$-Si (curve 5)

for crystalline silicon [11,12]. For the latter, relatively high values of the power factor can be obtained, however, the relatively high thermal conductivity for silicon leads to lowering of the ZT (formula 1) [11, 12].

The samples consisting of mostly unmixed Ge and Si particles (1-3) differ in the grain size (see Table 1), the porosity and the degree of Ge-Si intermixing. However, the thermoelectric parameters obtained for Samples 1-3 only slightly differ from one another. This can lead us to the conclusion that all three of the above content mentioned parameters do not exert a determining influence on both Seebeck coefficient and electrical conductivity. We believe that Seebeck coefficient and electrical conductivity are mostly determined by the carriers transfer between the Si granules in the sintered material. The electrical conductivity through Ge grains is negligible because Ge particles do not form a closed path for current flow (Fig. 3(a-c)). Because of the hole type of conductivity for the initial Si source, the electrical conductivity of samples 1-3 is also determined by holes. The increase in the resistivity in the latter case, as compared with the original structure $(p-\mathrm{Si})$, is attributed to the influence of the internal barriers between grain boundaries, as well as with the carried depleted regions on the $\mathrm{Si}-\mathrm{Ge}$ interfaces that are formed due to different types of doping (by analogy with space charge regions at the border of $p$ - $n$ junctions [13]).

The magnitude of the Seebeck coefficient for the samples with unmixed Ge and Si grains is apparently determined by Si granules, as is shown by the sign of the Seebeck voltage. The difference between $\alpha$ values for Samples 1-3 is insignificant; such difference can be attributed to the experimental error and to the difference between the resistivity values. It is known that the Seebeck coefficient value for a material increases with increased resistivity [1].

From such point of view, the variation of the grain parameters, the porosity and Ge-Si mixing in samples 1-3 only lead to some small changes of thermoelectric properties. For example, sample 1 is a denser material, in which a greater number of boundaries between $\mathrm{Ge}$ and $\mathrm{Si}$ grains are formed and the effect of depleted regions on the electrical resistivity is higher. For this sample, slightly greater resistivity value was obtained as compared with the Samples 2 and 3 (Fig. 4, curve 1).

A fundamentally different character of the temperature dependences of thermoelectric coefficients was revealed in Sample 4. For this sample, the mixing of Ge and Si atoms was confirmed by both the XPS phase analysis and the energy-dispersive spectroscopy analysis. The crystalline structure is not determined by the initial grain size and a certain decrease in the crystalline quality was revealed (Fig. 1). The influence of the new crystal structure on the thermoelectric properties is hard to derive because the most significant influence on Seebeck coefficient and conductivity is exerted by the carrier concentration. Because of the much greater doping level of the original Ge material (about $10^{18} \mathrm{~cm}^{-3}$ donors as compared with $2 \times 10^{15} \mathrm{~cm}^{-3}$ acceptors in $\mathrm{Si}$ ), the acceptor impurity from Si was compensated and, accordingly, the sign of the conductivity was changed; greater doping levels also provided lower resistivity values. In addition, one can suggest that the absence of internal $p$ - $n$ junctions in Sample 4 additionally reduces the resistivity value.

We emphasize that the use of the initial Ge and Si materials with different types of doping makes it possible to determine the degree of mixing of $\mathrm{Ge}$ and $\mathrm{Si}$ in the sintered material by the measuring the evolution of the resistivity. In the case of effective mixing, conductivity is determined by the material with a higher level of doping.

The lower absolute value of the Seebeck coefficient revealed for Sample 4, as compared with the one for the rest of the samples, is explained by: 
(1) lower resistivity of Sample 4. The dependence of $\alpha$ on the resistivity of a semiconductor material is a well-known fact [1];

(2) change of the type of conductivity (the electrons are characterized by the greater mobility as compared with holes);

(3) change of the grain structure type and the intrinsic properties of the material (a $\mathrm{Si}_{1-x} \mathrm{Ge}_{x}$ solid solution was obtained of instead of isolated Ge and Si grains).

In addition, we also note the increase in the power factor of the new polycrystalline solid solution as compared with the initial $p$-Si material. The latter is simply due to the greater doping level of the fabricated solid solution, such increase is also a well-known fact [1].

In conclusion, in the presented paper, the initial stages of Ge and Si nanopowder sintering during the fabrication of thermoelectric materials were studied. It was shown that in the first stage of sintering, there is no significant mixing of the $\mathrm{Ge}$ and $\mathrm{Si}$ atoms, and thermoelectric properties mostly depend on the carrier transport through $\mathrm{Si}$ grains in the sintered material. As the sintering process continues, Ge and $\mathrm{Si}$ are mixed, which results in the fabrication of a new material with increased power factor value.

\section{Acknowledgements}

This work was supported by Russian Science Foundation (grant \#17-79-20173).

\section{References}

[1] Rowe Ed.D.M. Termoelectric hand book macro to nano. CRC Press, Boca Raton, 2006.

[2] Hicks L., Dresselhaus M. Thermoelectric figure of merit of a one-dimensional conductor. Physical Revew B, 1993,47, P. 16631-16634.

[3] Minnich A.J., Dresselhaus M.S., Ren Z.F., Chen G. Bulk Nanostructured Thermoelectric Materials: Current Research and Future Prospects. Energy \& Environmental Science, 2009, 2 (5), P. 466-479.

[4] Cook B.A., Harringa J.L., Han S.H., Vining C.B. $\mathrm{Si}_{80} \mathrm{Ge}_{20}$ thermoelectric alloys prepared with GaP additions. J. Appl. Phys., 1995, 78, P. $5474-5480$

[5] Cook B.A., Harringa J.L., Han S.H., Beaudry B.J. Parasitic effects of oxygen on the thermoelectric properties of $\mathrm{Si}_{80} \mathrm{Ge}_{20}$ doped with GaP and P. J. Appl. Phys., 1992, 72, P. 1423-1428.

[6] Lahwal A., Bhattacharya S., et al. Impact of yttria stabilized zirconia nanoinclusions on the thermal conductivity of n-type $\mathrm{Si}_{80} \mathrm{Ge}_{20}$ alloys prepared by spark plasma sintering. J. Appl. Phys., 2015, 117, 145101.

[7] Usenko A., Moskovskikh D., et al. Thermoelectric Properties of n-Type $\mathrm{Si}_{0.8} \mathrm{Ge}_{0.2}-\mathrm{FeSi}_{2}$ Multiphase Nanostructures. Scripta Materialia, 2017, 127, P. 63-69.

[8] Bathula S., Jayasimhadri M., Dhar A. Mechanical properties and microstructure of spark plasma sintered nanostructured p-type SiGe thermoelectric alloys. Materials \& Design, 2015, 87, P. 414-440.

[9] Zamanipour Z., Shi X., et al. The effect of synthesis parameters on transport properties of nanostructured bulk thermoelectric ptype silicon germanium alloy. Physica status solidi (a), 2012, 209 (10), P. 2049-2058.

[10] Dismukes, J.P., Ekstrom L., et al. Thermal and electrical properties of heavily doped Ge-Si alloys up to 1300 K. J. Appl. Phys., 1964, 35, P. 2899-2907.

[11] Weber L., Gmelin E. Transport properties of silicon. Applied Physics A, 1991, 53, P. 136-140.

[12] Boukai A.I., Bunimovich Y., et al. Silicon Nanowires as Efficient Thermoelectric Materials. Nature, 2008, 451, P. 168-171.

[13] Sze S.M., Ng Kwok K. Physics of Semiconductor Devices. John Wiley \& Sons. Hoboken, New Jersey, 2006,832 p. 